\title{
Variabilidad de los procesos selectivos para el acceso a las especialidades médicas en Europa: entre convergencia y divergencia
}

\author{
Nicola Lorusso, Beatriz González López-Valcárcel
}

Introducción. La formación médica en Europa después de la implantación del Espacio Europeo de Educación Superior (EEES) se enfrenta a nuevos desafíos, cambios de modelos y necesidad de adaptación a las nuevas prioridades de la sociedad.

Objetivo. Analizar y comparar los procesos de selección y las pruebas empleadas para acceder a una plaza de formación especializada en el ámbito europeo.

Materiales y métodos. Estudio descriptivo y comparativo basado en la búsqueda y análisis de información de libre acceso, publicada o disponible a través de Internet. La muestra incluye la mayoría de los países de la Unión Europea de los 27, los más influyentes en términos demográficos, con una alta capacidad para formar grandes números absolutos de profesionales de la salud.

Resultados. La selección para el acceso a las especialidades médicas puede clasificarse en local, regional, nacional y mixta, en función del proceso, e individualizada o generalizada, en función de las pruebas utilizadas. El perfil personal y profesional, valorado mediante una entrevista, es un elemento clave que muchos sistemas tienen en cuenta sobre todo en los procesos de selección locales. Los países que aplican un sistema de selección nacional emplean básicamente exámenes de opción múltiple.

Conclusiones. Más allá de las diferencias cruciales entre países de ámbito europeo, es fundamental insistir en la individualización de modelos integrados y asumibles, que evalúen de manera completa las competencias requeridas de la profesión médica y favorezcan una mayor armonización entre países.

Palabras clave. Espacio Europeo de Educación Superior. Especialidades médicas. Formación especializada.

Variability of selective processes for access to medical specialties in Europe: between convergence and divergence

Introduction. Following the implementation of the European Higher Education Area (EHEA), medical education in Europe faces new challenges, structural changes and a need to adapt to new priorities of society.

Aim. To analyze and to compare the process of selecting and the tests used to access specialized training places at European level.

Materials and methods. Descriptive and comparative study based on the search and analysis of information published or freely available on line. The sample includes most of EU-27, the most influential in terms of demographics, with a high capacity to form large absolute numbers of health professionals.

Results. The selection for access to medical specialties is outlined in local, regional, national and mixed according to the process, individualized or generalized according to the utilized evaluation types. The personal and professional profile, as assessed by an interview, is a key element that many systems have in mind especially in the local selection process. Countries which are implementing a national system used primarily multiple choice tests.

Conclusions. Beyond the crucial differences between countries at the European level, it is essential to insist on the individualization of integrated and realistic models, that comprehensively assess the competencies required of the candidates, and encourage greater harmonization between countries.

Key words. European Higher Education Area. Medical specialties. Specialized training.
Hospital Universitario Virgen del Rocío; Sevilla (N. Lorusso). Departamento de Métodos

Cuantitativos en Economía y Gestión; Universidad de Las Palmas de Gran Canaria; Las Palmas, España (B. González López-Valcárcel).

Correspondencia:

Dr. Nicola Lorusso. Hospital Universitario Virgen del Rocío. Avda. Manuel Siurot, s/n. E-41013 Sevilla.

E-mail:

n.lorusso@hotmail.com

Conflicto de intereses: No declarado.

Competing interests: None declared.

(c) 2013 FEM 


\section{Introducción}

Numerosos informes advierten que, en el mundo, los nuevos profesionales de salud no están siendo preparados para enfrentarse a los cambiantes desafíos de la sociedad [1-4]. A escala mundial [5-7], en la formación médica se está integrando un nuevo modelo basado en resultados (outcome-based education) [8], acorde con las nuevas prioridades sobre las que ha de basarse el perfil del médico del futuro. La implantación del Espacio Europeo de Educación Superior (EEES) ha representado un paso enorme para la convergencia europea en términos de reconocimiento de títulos. Sin embargo, el objetivo prioritario de armonizar la formación en Europa para favorecer la circulación o el intercambio de estudiantes y de profesionales está lejos de conseguirse [9]. Se insiste en la necesidad de plantear globalmente, y en el ámbito europeo, el reto de repensar en qué se está haciendo en el 'entrenamiento' de los profesionales de salud, qué se ha logrado, qué faltaría y qué se necesitaría hacer $[10,11]$. Mientras, todavía persisten las diferencias considerables en ciertas áreas de la educación sanitaria en todos los niveles: desde el pregrado hasta la formación continuada. El objetivo del estudio es analizar y comparar los procesos selectivos de acceso a las especialidades médicas en Europa, las diferentes pruebas aplicadas y sus adaptaciones a los nuevos modelos de formación.

\section{Materiales y métodos}

Estudio descriptivo basado en la búsqueda y análisis de información de libre acceso, publicada y disponible a través de Internet, teniendo en cuenta páginas oficiales con datos sobre las características investigadas. Se efectúa un análisis comparativo basado fundamentalmente en los países de la Unión Europea, describiendo el estado actual de las principales tendencias sin la necesidad de incluir a los 27 países que la conforman. Los criterios utilizados para justificar la elección de los países se deben a la necesidad de que la muestra sea lo suficientemente variada y representativa de la Unión Europea, con la posibilidad de obtener una particular visión sobre los países de reciente inclusión en 2004 y 2007. La muestra incluye los países europeos más influyentes en términos demográficos, con una alta capacidad para formar grandes números absolutos de profesionales de la salud. Se ha decidido incluir Irlanda por el relevante papel que desempeña en el ámbito de la migración de los profesionales de salud en Europa.

\section{Resultados}

Actualmente, las pruebas utilizadas en el contexto europeo como métodos de evaluación para el acceso a la formación de especializada son: preguntas escritas (especialmente las preguntas de opción múltiple), preguntas de respuesta abiertas y simulaciones escritas de un caso clínico, pruebas de examen clínico objetivo estructurado (ECOE) que incluyen las simulaciones por ordenador y los enfermos simulados estandarizados, lectura crítica de la literatura científica y entrevistas. La tabla aporta una visión general de las modalidades de evaluación y el tipo de pruebas en el proceso de selección en los diferentes países objetos de estudio. Las pruebas que se emplean varían desde una 'simple' entrevista (Austria, Alemania, Países Bajos, Polonia, Republica Checa y Suecia) o una prueba escrita de opción múltiple (España, Francia, Portugal, Italia, Rumania y próximamente Grecia), hasta procesos de mayor complejidad y más completos, como la ECOE [12] (Reino Unido e Irlanda). En Bélgica y Hungría, el proceso de selección y las pruebas empleadas varían en función de la especialidad elegida o la universidad de pertenencia. Algo parecido se puede afirmar de Reino Unido e Irlanda. La mayoría de los países valoran el currículo en el momento de la evaluación como recopilación de aquellos aspectos de la trayectoria formativa y laboral sobre los que se incidirá en la entrevista personal. De esta forma es posible clasificar los países en función del alcance de la prueba en dos tipos de sistema: sistema de evaluación individualizado (Alemania, Austria, Hungría, Irlanda, Republica Checa, Países Bajos, Polonia, Reino Unido, Suecia y Bélgica) y sistema de evaluación generalizado (España, Francia, Grecia, Italia, Portugal, Rumania). Al mismo tiempo, en función del tipo de proceso, se perfilan diferentes tipos de sistemas de reclutamiento: local (Italia, Alemania, Suecia, Austria, República Checa, Bélgica, Polonia, Hungría y Países Bajos), regional (Rumania y Francia), nacional (España, Portugal, Grecia) y mixto (Irlanda y Reino Unido). Los países mediterráneos proponen un examen de entrada con distintas características, por lo general, de opción múltiple. Los países anglosajones aplican sistemas de evaluación integrados e individualizados, y los países nórdicos y centroeuropeos fundamentan el proceso en la entrevista, que se basa, como en el sistema anglosajón, en un sistema de solicitud individualizado dirigido a los distintos servicios acreditados, cuya regulación y garantía de derechos varían mucho entre los distintos países y centros [13]. 


\section{Discusión}

El análisis de las diferentes modalidades de acceso a las especialidades médicas en algunos países de $\mathrm{Eu}$ ropa destaca la falta de un proceso estandarizado para la selección de los residentes. En muchos países, la formación de posgrado está experimentando cambios requeridos o recomendados. Las razones dadas para justificar los cambios incluyen [3]:

- Superación del modelo tradicional hacia el modelo basado en resultados.

- Respuestas a las crecientes presiones políticas, económicas, sociales y tecnológicas en el sistema educativo.

- Necesidad de un sistema de formación más sostenible.

- Importancia de una elección 'vocacional' de la carrera profesional por parte de los residentes.

Se evidencia que los procesos de selección no siempre se sustentan sobre una base objetiva que abarque conocimiento, actitudes y habilidades en su conjunto. En los países donde la entrevista es la base para la selección o se realiza localmente, se corre el riesgo de que el acceso recaiga en la decisión de los que influyen en el proceso de selección, incumpliendo los niveles satisfactorios de igualdad, objetividad e imparcialidad. El proceso local presenta ventajas en términos de flexibilidad en la selección, permitiendo un contacto más directo entre hospitales/ universidades, seleccionadores y candidatos en la evaluación de competencias personales y comunicativas, aunque puede crear 'círculos viciosos' al favorecer a médicos que, por posición social, económica o política, tienen mejor relación con el mundo médico-académico. Se trata de un proceso que podría no garantizar la igualdad de oportunidades para los solicitantes, sobre todo cuando no cumplen los estándares y los criterios objetivos.

Los países con un sistema centralizado (nacional o regional) garantizan un sistema de acceso más objetivo, uniforme, igualitario, con una limitada manipulación por parte de los evaluadores de los resultados, obtenidos éstos mediante las pruebas de selección, lo que garantiza una selección estandarizada. No obstante, como ocurría también en los procesos locales, aunque por otras razones, las asignaciones pueden no ajustarse a la vocación de cada aspirante [14]. Este modelo plantea la problemática de la escasa validez que ofrece para la evaluación de las competencias y otras habilidades adquiridas durante la carrera de medicina, ya que el sistema de acceso está diseñado para medir principalmente conocimiento y datos concretos [15].
Tabla. Diferentes pruebas utilizadas en el proceso de selección para el acceso a la formación especializada en los países analizados.

\begin{tabular}{|c|c|c|c|c|c|c|c|}
\hline & Currículo & $\begin{array}{l}\text { Expediente } \\
\text { académico }\end{array}$ & $\begin{array}{l}\text { Examen } \\
\text { de opción } \\
\text { múltiple }\end{array}$ & $\begin{array}{c}\text { Examen de } \\
\text { respuesta } \\
\text { abierta }\end{array}$ & ECOE & $\begin{array}{l}\text { Lectura } \\
\text { crítica de } \\
\text { artículos }\end{array}$ & Entrevista \\
\hline Alemania & $\bullet$ & & & & & & $\bullet$ \\
\hline Austria & $\bullet$ & & & & & & $\bullet$ \\
\hline Bélgica & • & • & $\cdot /-$ & $\cdot /-$ & & & $\bullet$ \\
\hline España & & • & $\bullet$ & & & & \\
\hline Francia & & & & $\bullet$ & & $\bullet$ & \\
\hline
\end{tabular}

Grecia

\begin{tabular}{lcccc}
\hline Hungría & $\bullet /-$ & $\bullet /-$ & $\bullet /-$ & $\bullet /-$ \\
\hline Irlanda & $\bullet$ & $\bullet /-$ & $\bullet$ \\
\hline Italia & $\bullet$ & $\bullet$ & $\bullet$ & $\bullet$
\end{tabular}

\begin{tabular}{llll}
\hline Países Bajos & $\bullet$ & & $\bullet$ \\
\hline Polonia & $\bullet$ & $\bullet$ & $\bullet$ \\
\hline
\end{tabular}

\begin{tabular}{lllll}
\hline Portugal & $\bullet$ & $\bullet$ & $\bullet$ \\
\hline Rep. Checa & $\bullet$ & $\bullet$ & $\bullet$ \\
\hline Rumania & $\bullet$ & $\bullet$ & $\bullet$ \\
\hline Reino Unido & $\bullet$ & & \\
\hline Suecia & $\bullet$ & & \\
\hline
\end{tabular}

- simboliza la utilización de la prueba; •/- simboliza que la prueba puede o no utilizarse según el tipo de especialidad. ECOE: examen clínico objetivo estructurado.

Cabe destacar que cada prueba, en su individualidad, carece de la riqueza asociada a la reforma de la educación médica, sobre valores profesionales, actitudes y comportamientos, liderazgo y habilidades de comunicación. En esta óptica, la combinación de diferentes técnicas para medir distintas competencias parece fundamental en la evaluación de los residentes y no la limitan a específicos campos de formación, permitiendo adaptarse a las nuevas funciones y valores de los médicos que se están formando para el futuro [16].

La selección de los médicos para la formación de posgrado en el ámbito europeo tendría que adecuarse en muchos aspectos para lograr una formación integrada del médico en una 'nueva visión de for- 
Figura. Principios para nuevos modelos de selección de los médicos.

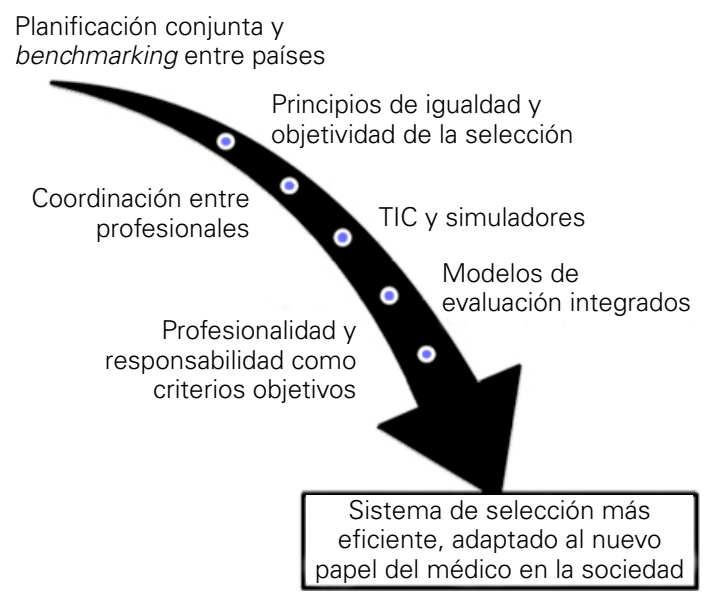

mación', basada en el e-learning, simuladores para el aprendizaje de habilidades manuales, autoevaluación y evaluación de competencias éticas y morales, en un proceso mixto, cognitivo-emocional [17].

En el contexto europeo podría fomentarse un proceso de selección que alcance un cierto grado de armonización y transparencia, acorde con los estándares globales en la educación médica de posgrado [18]. Asimismo, se podrían aprovechar los sistemas actuales o implantar nuevos, sobre una base de objetivos, criterios y tipos de pruebas flexibles, medibles y comparables, para poder evaluar los resultados conseguidos [19], favorecer el benchmarking entre países y consolidar el EEES (Figura).

En conclusión, existen diferencias cruciales en las etapas de acceso a las especialidades médicas en Europa y es deseable fomentar una mayor armonización entre modelos en el ámbito europeo para apoyar la transición entre etapas y maximizar el aprendizaje de manera igualitaria. Hay que adaptar las competencias basadas en objetivos al nuevo papel del médico en la sociedad a través de modelos integrados capaces de abarcar ampliamente co- nocimiento, habilidades y actitudes de la profesión médica.

Bibliografía

1. Pardell-Alentà $\mathrm{H}$, coord. El médico del futuro. Barcelona: Fundación Educación Medica, 2009.

2. General Medical Council. Tomorrow's doctors. 3 ed. London: GMC; 2009.

3. Hodges BD. A tea-steeping or i-Doc model for medical education? Acad Med 2010; 85: S34-44.

4. Frenk J, Chen L, Bhutta ZA, Cohen J, Crisp N, Evans T, et al. Health professionals for a new century: transforming education to strengthen health systems in an interdependent world. Lancet 2010; 376: 1923-58.

5. Simpson JG, Furnace J, Crosby J, Cumming AD, Evans PA, Friedman Ben David M, et al. The Scottish doctor -learning outcomes for the medical undergraduate in Scotland: a foundation for competent and reflective practitioners. Med Teach 2002; 24: 136-43.

6. CanMEDS 2000. Extract from the CanMEDS 2000 Project Societal Needs Working Group Report. Med Teach 2000; 22: 549-54.

7. Accreditation Council for Graduate Medical Education. ACGME Outcome Project 1999. URL: http://www.acgme.org.

8. Harden RM. Outcome-based education: the future is today. Med Teach 2007; 29: 625-9.

9. Lobato RD, Lagares A, Alén JF, Alday R. El desarrollo del proceso de Bolonia y el Grado de Medicina. Situación actual y expectativas para su implantación definitiva. Neurocirugía 2010; $21: 146-56$.

10. Oriol-Bosch A. De Flexner a Bolonia. Educ Med 2010; 13: 193-5.

11. García PJ. Repensando la educación de los profesionales de salud del siglo xxI: cambios y acciones en un mundo global. Rev Peru Med Exp Salud Pública 2011; 28: 390-9.

12. Yang YY, Lee FY, Hsu HC, Huang CC, Chen JW, Lee WS, et al. A core competence-based objective structured clinical examination (OSCE) in evaluation of clinical performance of postgraduate years-1 (PGY1) residents. J Chin Med Ass 2011; 74: 198-204.

13. Morán JM, San Emeterio E. Las especialidades médicas en Europa. Organización y desarrollo. Educ Med 2005; 8: 120-7.

14. Lindgren S, Gordon D. The doctor we are educating for a future global role in health care. Med Teach 2011; 33: 551-4.

15. Martínez-Carretero JM. Los métodos de evaluación de la competencia profesional: la evaluación clínica objetiva estructurada (ECOE). Educ Med 2006; 8 (Suppl 2):18-22.

16. Spiegel W, Pichlhöfer O, Haoula D, Schneider B, Maier M. Specialty selection and relative job satisfaction of family physicians and medical specialists in Austria. Croat Med J 2008; 49: 375-83.

17. Tizón JL. Sobre la formación integral del médico: aportaciones de las primeras jornadas estatales de estudio y reflexión sobre el tema. Educ Med 2009; 12: 209-21.

18. World Federation of Medical Education. Postgraduate medical education. WFME global standards for quality improvement. Copenhagen, March 2003. URL: http://www.wfme.org.

19. Mortensen L, Malling B, Ringsted C, Rubak S. What is the impact of a national postgraduate medical specialist education reform on daily clinical training 3.5 years after implementation? A questionnaire survey. BMC Med Educ 2010; 10: 46. 